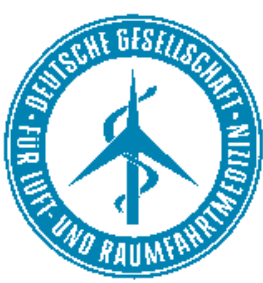

Mitteilungen der

Deutschen Gesellschaft für

Luft- und Raumfahrtmedizin e. V. Nr. 4/2015

DGLRM-Homepage: www.dglrm.de

Geschäftsstelle der DGLRM

Christine Gens

Postfach 870204

13162 Berlin

E-Mail: christine.gens@dglrm.de

Tel.: 0176/5624-2876

\section{Wir begrüßen als neue Mitglieder}

- Nurcihan Joy Koc aus Viersen

- Sven Kühn aus Fürstenfeldbruck

- Steffen Kalina aus Köln

- Dr. Klaus Becker aus Düsseldorf

\section{Wir gratulieren}

- 65 Jahre, Dr. Andrew Bellenkes,

Pinswang, Österreich, 21.07.1950

\section{Verantwortlich für den Inhalt}

\section{der DGLRM-Seiten}

Deutsche Gesellschaft für Luft- und Raumfahrtmedizin e. V. (DGLRM) OFA PD Dr. Carla Ledderhos, Präsidentin Zentrum für Luft- und Raumfahrtmedizin der Luftwaffe

Str. der Luftwaffe 322

82242 Fürstenfeldbruck

carlaledderhos@bundeswehr.org

Redaktionsschluss: 15.07.2015

Bankverbindung

Deutsche Bank Hamburg

Konto-Nr.: 388948200

BLZ: 20070000

IBAN: DE 63200700240388948200

BIC: DEUTDEDBHAM

Änderungen für die Mitgliederkartei bitte an: Dipl. Ing. Christine Gammel

Zentrum für Luft- und Raumfahrtmedizin

der Luftwaffe

Str. der Luftwaffe 322

82242 Fürstenfeldbruck

christinegammel@bundeswehr.org

\section{Sehr geehrte Damen und Herren, liebe DGLRM-Mitglieder,}

am 27. Juni hat die 32. Bayerische, 25. Baden-Württembergische und 22 . Sächsische Fliegerärztetagung in altbewährter Weise unter der Leitung von Dr. Peter Frank aus Gröbenzell und Prof. Dr. Heinz Jürgen Deuber aus Bamberg in Fürstenfeldbruck stattgefunden. Mit 112 sucht. Abwechslungsreich war auch das Programm. Mit Themen aus der Ophthalmologie, Inneren Medizin, Orthopädie, Neurologie und Psychiatrie sowie vielen Fallbesprechungen war das behandelte Spektrum sehr breit gefächert und hielt für jeden Teilnehmer die eine oder andere Neuigkeit bereit. Updates zu Impfungen sowie Fliegertauglichkeit für T2DM, KHK, Asthma / COPD und NOAKS sowie der Vortrag aus dem Referat Flugmedizin des LBA haben das Treffen der Flugmediziner abgerundet und viel Gesprächsstoff geboten. Die Veranstaltung wurde mit Unterstützung des Zentrums für Luft- und Raumfahrtmedizin der Luftwaffe, der DGLRM und des DFV durchgeführt und mit 7 Fortbildungsstunden vom LBA und mit 11 Punkten der Kategorie A der Bayerischen Landesärztekammer anerkannt.

Vorbereitungen zur AsMA-Tagung 2016 Die 87. Tagung der Aerospace Medical Association (AsMA), die vom 24. bis 28. April 2016 in Harrah's Resort, Atlantic City, NJ, USA stattfindet, will auch von unserer Gesellschaft wieder gut vorbereitet sein. Es ist bereits ein Aufruf per E-Mail an unsere Mitglieder versandt worden, um für Beiträge für das deutschsprachige Panel zu werben. Erfreulicherweise hat auch schon Teilnehmern war sie wieder sehr gut be- eine Reihe von Kollegen ihr Interesse an einer aktiven Teilnahme bekundet und ihre Vortragsabsichten bei Herrn PD Dr. Jochen Hinkelbein angemeldet. Dafür bedanken wir uns ganz herzlich! Dennoch können immer noch Wünsche berücksichtigt werden. Sollten Sie also auch vorhaben, einen Beitrag anzumelden, zögern Sie bitte nicht, dies (so bald als möglich) zu tun. Wenn wir genügend Interessenten finden, sollen, wie auch bereits in diesem Jahr, eine deutschsprachige und eine englischsprachige Sitzung eingereicht werden.

Internationales Symposium

„Violence, attacks and aggression in transportation"

Vom 16. bis 18. September 2015 findet in Hamburg ein internationales Symposium mit Workshop zum Thema „Violence, attacks and aggression in transportation“ statt. Hier sollen auch Übergriffe im Luftverkehr thematisiert werden. Das vorläufige Programm zu dieser Veranstaltung finden Sie am Ende dieses Artikels. Für Mitglieder der DGLRM ist die Teilnahme frei. Bitte melden Sie sich bei Herrn Dr. Jörg Hedtmann (joerg.hedtmann@bgverkehr.de) an.

\section{Jahrestagung 2015}

Die Vorbereitungen zu unserer Jahrestagung laufen jetzt auf Hochtouren. Bei den bisher eingegangenen Anmeldungen zur Jahrestagung ist ein besonderes Interesse der Teilnehmer an den Fachbesichtigungen bei der BFU und beim DLR zu erkennen, während beim Niedersächsischen Zentrum für Luftfahrt und der Aerodata AG

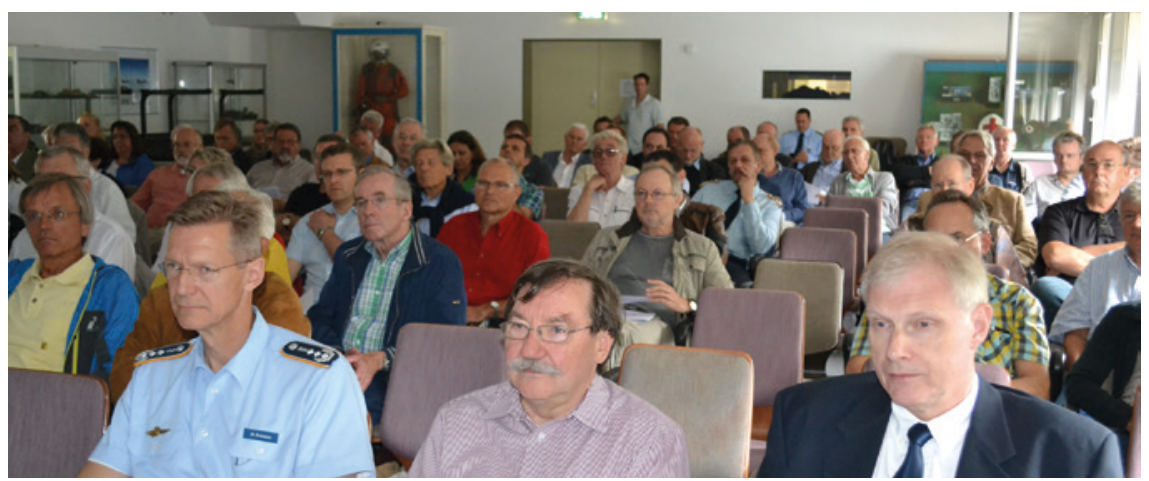

Abb. 1 Blick in den Hörsaal auf der 32. Bayerischen, 25. Baden-Württembergischen und 22. Sächsischen Fliegerärztetagung (1. Reihe v.I.n.r. OTA Dr. Bressem, Dr. Frank, Prof. Deuber). ～Quelle: Dr. Peter Frank 


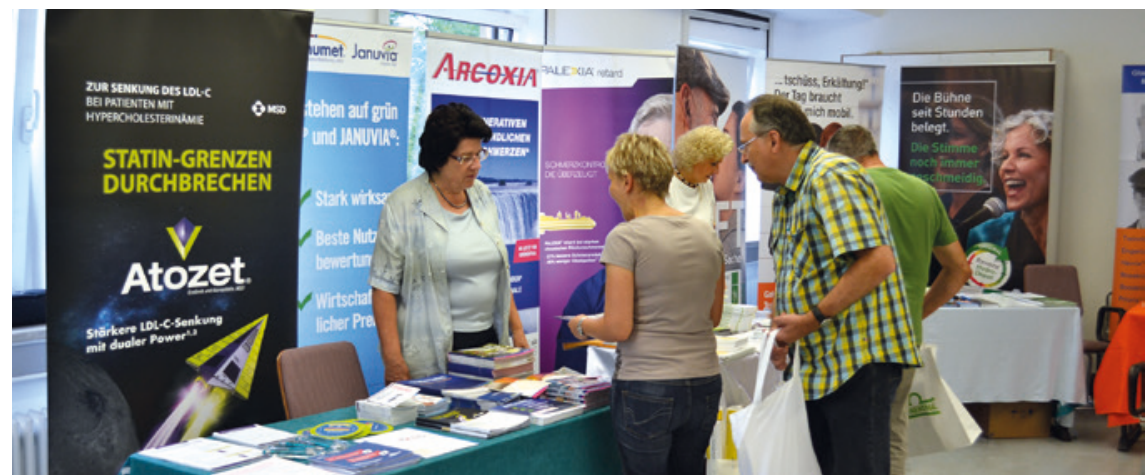

Abb. 2 Industrieausstellung auf der Fliegerärztetagung.

Quelle: Dr. Peter Frank

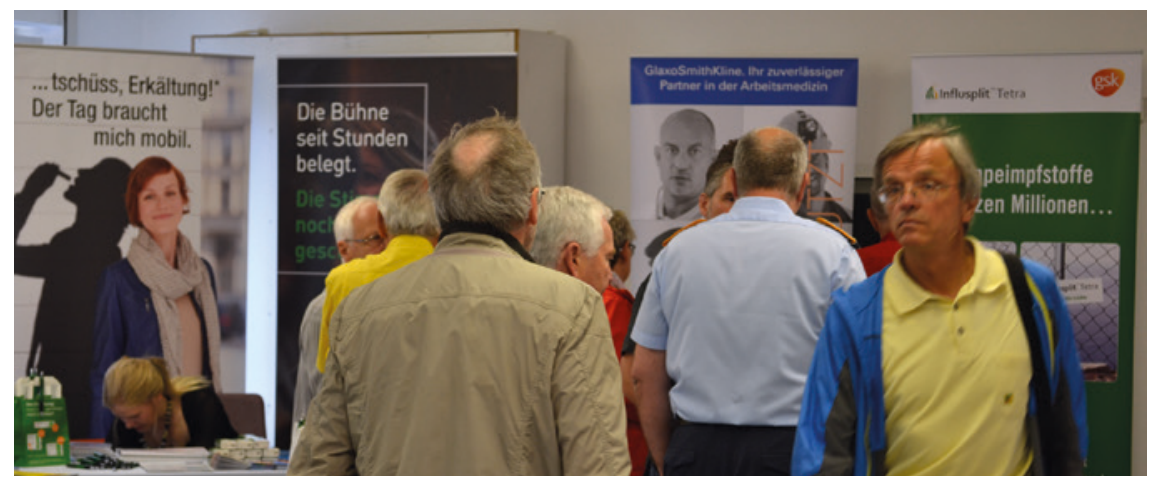

Abb. 3 Andrang bei den Ausstellern auf der Fliegerärztetagung in Fürstenfeldbruck. Quelle: Dr. Peter Frank

eher noch freie Valenzen vorhanden sind. Wir werden noch einmal Kontakt mit der Forschungsflughafen Braunschweig $\mathrm{GmbH}$ aufnehmen, um zu erkunden, ob dieser große Andrang insbesondere bei der BFU tatsächlich auch befriedigt werden kann. Sollte dies nicht möglich sein, werden wir die Anmeldungen in der Reihenfolge des Eingangs berücksichtigen müssen. Ich verspreche Ihnen, mein Möglichstes dafür zu tun, dass dieser Fall nicht eintritt.

Einladung zur Mitgliederversammlung 2015

am Freitag, 25.09.2015 um 17:00 Uhr

Ort: Hermann-Blenk-Saal des DLR am Forschungs-

flughafen Braunschweig

Tagesordnung:

TOP 1 Eröffnung und Begrüßung

TOP 2 Genehmigung der Tagesordnung

TOP 3 Bericht der Präsidentin

TOP 4 Bericht des Sekretärs

TOP 5 Bericht des Schatzmeisters

TOP 6 Bericht der Rechnungsprüfer

TOP 7 Entlastung des Vorstands

TOP 8 Berichte aus den Arbeitsgruppen

TOP 9 Zukunft der flugmedizinischen Aus- und Weiterbildung in Deutschland

TOP 10 Zeit und Ort der nächsten Mitgliederversammlung

TOP 11 Sonstiges
Bitte vergessen Sie nicht, sich rechtzeitig zur Jahrestagung anzumelden. Dies erleichtert nicht nur dem Vorstand die Planung, auch für Sie wird die Vorbereitung der Reise einfacher, da die Hotelkontingente uns nur bis zum 28. August eingeräumt worden sind. Danach müssen Sie sich Zimmer auf dem „freien Markt“ suchen, was im September in Braunschweig zum Problem werden könnte. Für weitere Informationen verweise ich auf unsere Homepage.

In der Hoffnung möglichst viele Teilnehmer auf unserer Jahrestagung begrüßen zu können, verbleibe ich mit den besten Wünschen für eine erholsame und anregende Sommerzeit

Ihre

Carla Ledderhos

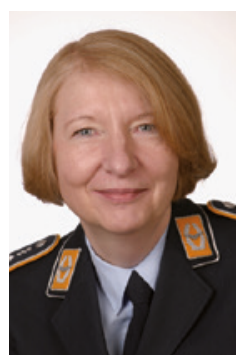

\section{Lehrgänge 2015}

23. Refresher-Seminar Flugmedizin (deutsch)

Dt. Akademie für Flug- und Reisemedizin $\mathrm{GmbH}$

Termin: 06.11.-08.11.2015

Ort: $\quad$ Lufthansa Training \& Conference Center, Seeheim-Jugenheim

Kontakt: Sigrid Froese, Tel: +49(0)69/69691222

Internet: www.flugmed.org/ www.eusam.org

EUSAM - Basic course 26 (englisch) European School of Aviation Medicine Termin: 03.09.-11.09.2016

Ort: Medizinischer Dienst der Deutschen Lufthansa auf der Lufthansa-Basis, Flughafen Frankfurt

Kontakt: Sigrid Froese, Tel: +49(0)69/69691222

Internet: www.flugmed.org/ www.eusam.org

EUSAM - Advanced course 26 (englisch)

European School of Aviation Medicine Termin: 03.12.-11.12.2016

Ort: $\quad$ Medizinischer Dienst der Deutschen Lufthansa auf der Lufthansa-Basis, Flughafen Frankfurt

Kontakt: Sigrid Froese, Tel: +49 (0)69/69691222

Internet: www.flugmed.org/ www.eusam.org

\section{Kongresse 2015}

Symposium „Gewalt, Übergriffe und Aggression im Transportwesen“ Termin: 16.09.-18.09.2015

Ort: Hamburg

$63^{\text {rd }}$ International Congress of Aviation and Space Medicine (ICASM)

Int. Academy of Aviation and

Space Medicine

Termin: 20.09.-24.09.2015

Ort: $\quad$ Oxford, UK

Internet: www.icasm2015.org

53. Jahrestagung der Gesellschaft für Luft- und Raumfahrtmedizin (DGLRM) Termin: 24.09.-26.09.2015

Ort: Forschungsflughafen Braunschweig

Internet: www.dglrm.de 